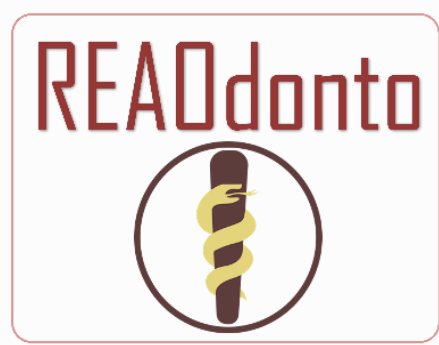

Revista Eletrônica Acervo
Odontológico

\section{ARTIGO ORIGINAL}

Recebido em: 5/2020

Aceito em: 6/2020

Publicado em: 8/2020

\title{
Avaliação da distância intercaninos e sua correlação com as más oclusões
}

\author{
Evaluation of intercanin distance and its correlation with maloclusions \\ Evaluación de la distancia intercanina y su correlación con malas oclusiones \\ Téssia Richelly Nóbrega Borja de Melo ${ }^{1 *}$, Gustavo Ramos Souza.
}

\begin{abstract}
Resumo: O objetivo deste trabalho é avaliar a correlação entre a distância intercaninos e as más oclusões. Tratou-se de um estudo observacional e prospectivo, cujos dados foram coletados de 110 documentações ortodônticas e modelos de estudo, de pacientes entre 12 e 35 anos. As variáveis estudadas foram: idade, sexo, padrão racial, relação molar de Angle, más oclusões e distância intercaninos. Os dados foram anotados em formulário específico e distribuídos em tabelas, onde aplicou-se a estatística analítica através dos testes t Student e ANOVA ( $p<0,05)$. Os resultados foram os seguintes: a média de idade foi de 17,7 anos; a maioria da amostra foi do sexo feminino $(58,7 \%)$ e de feodermas $(43,6 \%)$. A relação de Classe I apresentou maior frequência (46,4\% direita; $49,1 \%$ esquerda) e a má oclusão mais prevalente foi o apinhamento dentário $(67,7 \%)$. As médias das distâncias intecaninos foram: $33,98 \mathrm{~mm}$, para a arcada superior e $26,11 \mathrm{~mm}$ para a interior. A distância intercaninos na arcada superior de homens, foi estatisticamente maior que nas mulheres; assim como os arcos inferiores de jovens de 12 a 20 anos, foram maiores do que os de adultos de 21 a 35 anos. Concluiu-se que não houve correlação significativa entre a distância intercaninos e as más oclusões.
\end{abstract}

Palavras-chave: Arco dental, Dente canino, Ortodontia.

Abstract: The objective of this work is to evaluate a correlation between channels at a distance and as more occlusions. It treated an observational and prospective study, data that were collected from 110 orthodontic documents and study models, from patients between 12 and 35 years old. The variables studied were: age, sex, racial pattern, molar angle ratio, plus occlusions and intercanine distances. The data were recorded in specific forms and distributed in tables, where analytical statistics were applied through Student and ANOVA tests $(p<0.05)$. The results were as follows: the average age was 17.7 years; a majority of the sample was female $(58.7 \%)$ and feoderms (43.6\%). The Class I relationship is more frequent $(46.4 \%$ on the right; $49.1 \%$ on the left) and a more prevalent occlusion was tooth crowding (67.7\%). The distance media were: 33.98 $\mathrm{mm}$, for an upper arch and $26.11 \mathrm{~mm}$ for an interior. The intercanine distance in the upper arch of men was statistically greater than in women; as well as children under 12 to 20 years old, those over 21 to 35 years old. It was concluded that there was no significant correlation between intercanine distances and as more occlusions.

Keywords: Dental arch, Canine tooth, Orthodontics.

Resumen: El objetivo de este trabajo es evaluar una correlación entre canales a distancia y como más oclusiones. Se trató un estudio observacional y prospectivo, datos recopilados de 110 documentos de ortodoncia y modelos de estudio, de pacientes entre 12 y 35 años. Las variables estudiadas fueron: edad, sexo, patrón racial, relación de ángulo molar, más oclusiones y distancias intercaninas. Los datos se

${ }^{1}$ Centro Universitário de Patos (UNIFIP). Patos - Paraíba. *E-mail: tessiaborja@yahoo.com.br 
registraron en formas específicas y se distribuyeron en tablas, donde se aplicaron estadísticas analíticas mediante pruebas de Student y ANOVA $(p<0.05)$. Los resultados fueron los siguientes: la edad promedio fue de 17.7 años; La mayoría de la muestra eran mujeres (58.7\%) y feodermos (43.6\%). La relación de Clase I es más frecuente (46.4\% a la derecha; $49.1 \%$ a la izquierda) y una oclusión más frecuente fue apiñamiento dental (67.7\%). Los medios de distancia fueron: $33.98 \mathrm{~mm}$, para un arco superior y $26.11 \mathrm{~mm}$ para un interior. La distancia intercanina en el arco superior de los hombres fue estadísticamente mayor que en las mujeres; así como niños menores de 12 a 20 años, mayores de 21 a 35 años. Se concluyó que no había una correlación significativa entre las distancias intercaninas y a medida que aumentaban las oclusiones.

Palabras clave: Arco dental, Diente canino, Ortodoncia.

\section{INTRODUÇÃO}

A oclusão é parte integrante do sistema mastigatório. É a área da odontologia reservada ao estudos das relações de contatos dentários entre os arcos superior e inferior, e suas implicações sobre as demais estruturas bucais: gengiva, ossos, músculos, ligamentos e articulações temporomandibulares. A oclusão pode variar entre indivíduos de acordo com o tamanho e a conformação dos dentes; as posições dentárias e sequência de erupção; a forma e o tamanho dos arcos dentários; e o padrão de crescimento crânio-facial; além de influências do meio ambiente, modificações funcionais e possíveis patológicas associadas (ARAÚJO SCCS, et al., 2014).

O conceito de oclusão evoluiu de uma concepção meramente estática, onde era considerado apenas o contato entre os dentes, cúspide-fossa; para uma conceituação dinâmica, incluindo-se dentes e estruturas vizinhas, tais como: músculos, articulações, planos inclinados e bases ósseas. As fases da oclusão dependem da posição de contato ou afastamento dos dentes, da contração ou relaxamento dos músculos mastigadores e da movimentação ou imobilização da mandíbula (PERREIRA JCM, et al., 2012).

As más oclusões representam desvios de normalidade dos arcos dentários, do esqueleto facial ou de ambos, com reflexos variados tanto nas diversas funções do aparelho mastigador, quanto na aparência e na autoestima dos indivíduos afetados. A incidência de casos de má oclusão tem aumentado progressivamente, alcançando um número preocupante, o que as torna o terceiro problema de saúde bucal pública no mundo, sendo precedida apenas pela cárie dentária e pela doença periodontal (SOUSA JP e SOUSA SA, 2013).

A correção das más oclusões e das bases ósseas, coloca os dentes em equilíbrio e posições ideais, harmonizando o sistema estomatognático. Há uma melhora na estética e na função desde que se tenham saudáveis as estruturas de suporte e periodontais. Um planejamento preciso e de mecânica bem empregada, proporcionarão sucesso e estabilidade em longo prazo (GUILLEN AGLE, et al., 2020).

Para isso, é importante a observação da morfologia dos arcos dentários de cada paciente, pois o respeito à sua individualidade evitará problemas periodontais como retrações gengivais, instabilidade nas movimentações dentárias e pouca eficiência nos resultados estéticos alcançados durante a terapia ortodôntica (TRIVIÑO T, et al., 2007; RODRÍGEZ AB et al., 2016).

Cada indivíduo possui uma disposição dentária particular. Os seres humanos têm os dentes dispostos entre si formando os arcos, os quais apresentam características individuais importantes que são submetidas às funções fisiológicas. Análises estatísticas mostram que as dimensões dos arcos dentários em diferentes etnias tem uma menor influência ambiental e são controladas, preferencialmente, por fatores genéticos (ALKHATEEB SN, et al., 2006; MASPERO C, et al., 2020).

As distâncias intercaninos e intermolares são estabelecidas aos oito anos de idade, depois da irrupção dos incisivos, com aumento mínimo até a dentadura permanente se completar, ocorrendo por volta dos 13 anos. $\mathrm{Na}$ idade adulta ocorre uma redução dos comprimentos dos arcos superior e inferior, além da diminuição da distância intercaninos e aumento da distância intermolares (HENRIKSON J, et al., 2001; BONDEVIK O, 2007). 
A distância intercaninos é a medição da largura entre os caninos no arco dentário. É medida da ponta de cúspide ou do centro da faceta de desgaste onde possivelmente era a cúspide; do canino de um lado do arco, ao canino contralateral. A distância normal entre os caninos superiores varia de $25 \mathrm{a} 40 \mathrm{~mm}$. Medidas menores que $25 \mathrm{~mm}$ podem ser identificadas como de uma arcada infantil. Aquelas entre 25 e $30 \mathrm{~mm}$, podem descritas como de um adulto pequeno (KANASHIRO LK e VIGORITO JW, 2007; GUILLEN AGLE, et al., 2020).

Angle $E$ (1907) difundiu o conceito de que para se chegar a uma oclusão ideal, todos os dentes deveriam estar presentes na arcada, levando muitos profissionais a realizarem expansões acentuadas dos arcos dentais durante o tratamento ortodôntico e, como consequência, ocorriam recidivas, como apinhamentos, que eram justificadas pela falha no alcance à oclusão ideal ao final da terapia ortodôntica.

O apinhamento dentário ocorre principalmente devido ao tamanho do dente versus a discrepância no comprimento do arco. Além disso, a estabilidade pós-ortodôntica depende muito da manutenção da forma do arco inferior; assim, as dimensões do arco dentário são valores importantes a serem investigados (MASPERO C, et al., 2020).

Segundo Sánchez RMP et al. (2019), para a manutenção do equilíbrio muscular, é importante a preservação da distância intercaninos original do arco mandibular. Além disso, declararam que os caninos representam as pedras fundamentais ou os alicerces dos arcos dentais, reafirmando a importância, durante o tratamento ortodôntico, da conservação da distância intercaninos.

Bondevik O (2007) afirmou que a largura transversal medida de um dente canino ao outro na dentição inferior representaria um índice preciso do equilíbrio muscular ao indivíduo e limites para a expansão da dentição nesta área durante tratamento. Para manter uma condição de equilíbrio, os músculos periorais desempenhariam papel fundamental.

Segundo Patricia PR et al. (2007), é produzido um deslocamento leve, mas constante, dos caninos (superiores labialmente e os inferiores lingualmente) em arcos mais estreitos, associados a extrusão dos caninos superiores e inferiores e a movimentos laterais da mandíbula, durante a mastigação. Esse deslocamento, é fisiológico, e ocorrerá desde que haja equilíbrio muscular no paciente.

Entretanto, nas movimentações ortodônticas, as distâncias entre caninos inferiores, e consequentemente superiores, são consideradas determinantes na largura da arcada dental. Portanto, alterações consideráveis nessas distâncias, principalmente em sentido vestibular dos incisivos, deverão ser bem direcionadas e controladas, para manter a estabilidade após o término da ortodontia fixa (KANASHIRO LK, VIGORITO JW, 2007; GUILLEN AGLE, et al., 2020).

Frente ao exposto, o objetivo do presente trabalho é avaliar a distância intercaninos e correlacioná-la às más oclusões.

\section{MÉTODOS}

O presente trabalho seguiu os preceitos da Bioética (Resoluções 466/12 e 510/16) e foi aprovado pelo Comitê de Ética em Pesquisa do Centro Universitário de Patos UNIFIP/PB, sob o parecer consubstanciado número: 3.170.033/ 2019. Realizou-se um estudo observacional e prospectivo, cuja amostra foi composta por 110 documentações ortodônticas, pertencentes ao acervo do curso de especialização em Ortodontia do Centro Universitário de Patos - UNIFP/PB.

As variáveis estudadas foram as seguintes: distância intercaninos, relação molar segundo a classificação de Angle (Classe I - cúspide mésio-vestibular do molar superior oclui entre a cúspide mésio-vestibular e a mediana do molar inferior; Classe II - cúspide mésio-vestibular do molar superior oclui à frente da cúspide mésio-vestibular do molar inferior; Classe III - cúspide mésio-vestibular do molar superior oclui atrás da cúspide mésio-vestibular do molar inferior); e más oclusões (mordida aberta; mordida cruzada e apinhamento dentário); além da caracterização da amostra por meio do padrão racial, idade e sexo. Todas foram coletadas por um único examinador, treinado e calibrado. 
Foram incluídos aleatoriamente, exames de homens e mulheres, nas faixas etárias de 12 a 35 anos, desde que os exames fossem de boa qualidade, quanto à nitidez e polimento adequado os modelos de estudo; assim como assinados pelos pacientes ou responsáveis. Foram excluídos exames mal executados, com modelos fraturados ou com baixa qualidade no acabamento desses modelos.

Para a medição da distância intercaninos, a amostra foi dividida em 64 modelos do sexo feminino e 46 do masculino. Utilizando-se como pontos de referência a ponta de cúspide dos caninos ou os pontos médios das facetas de desgaste, em cada modelo de gesso de ambos arcos, maxilar e mandibular, posicionou-se o paquímetro digital eletrônico (MTX ${ }^{\circledR}$ com precisão $0,01-150 \mathrm{~mm} / 0,0005$ ") sobre essas superfícies, obtendo-se as medições das distâncias. As numerações foram anotadas no formulário para coleta de dados. A cada medição o paquímetro foi recalibrado na zeragem do instrumento (Figura 1). (ANDREWS LF, 1972; KANASHIRO LK, VIGORITO JW, 2007).

Figura 1 - Paquímetro Digital MTX ${ }^{\circledR} 0,01-150$ mm/0,0005" para medição da distância intercaninos.

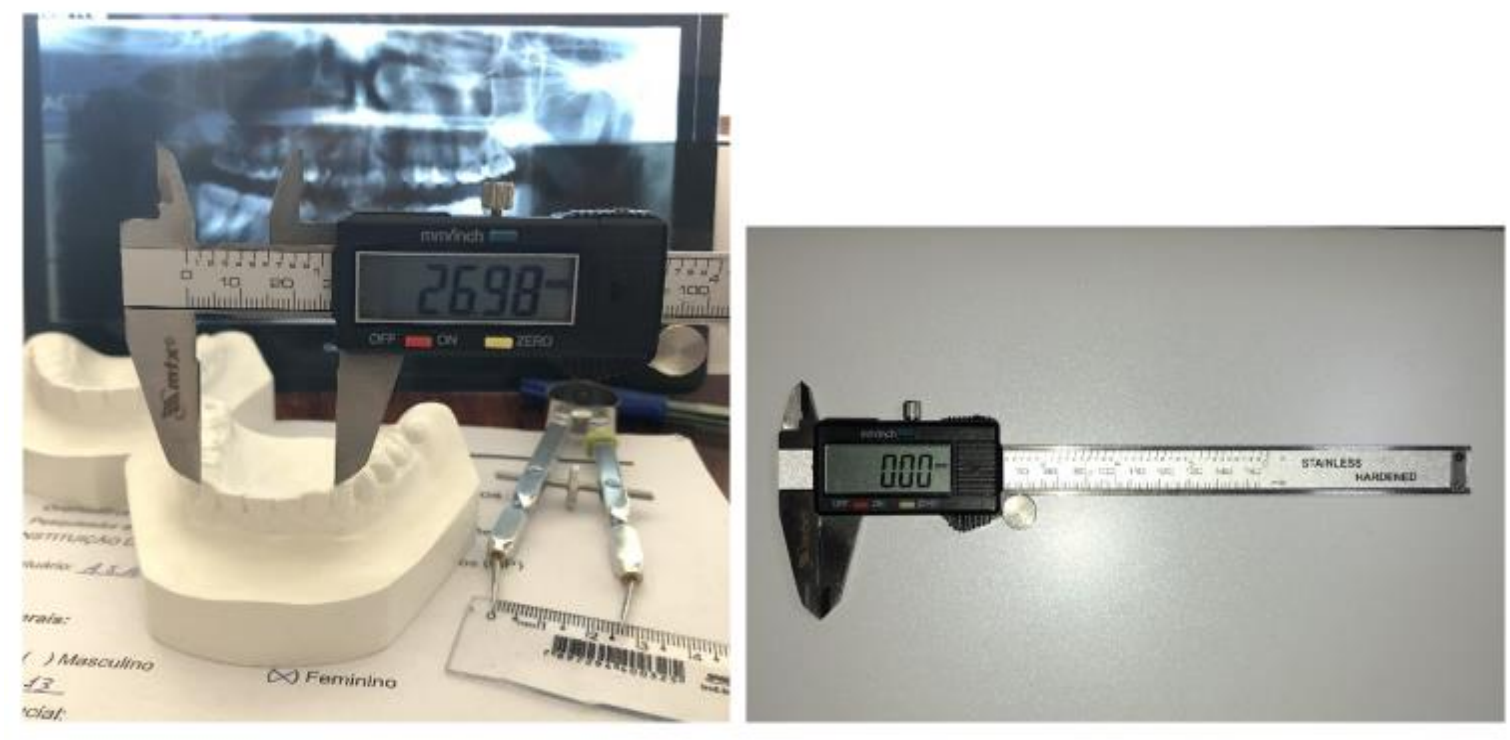

Fonte: Melo TRNB e Souza GR, 2020.

Quanto às demais variáveis: relação molar segundo a classificação de Angle, más oclusões, padrão racial, idade e sexo; todas foram coletadas pelo examinador, por meio da avaliação dos modelos de estudo e dos dados clínicos presentes nas documentações ortodônticas.

Todas as características referentes às variáveis estudadas foram anotadas em formulário específico confeccionado para tabulação dos dados. Posteriormente, as informações foram colocadas em tabelas e realizada a análise estatística de normalidade das variáveis quantitativas através do teste Kolmogorov Smirnov.

Em seguida, para comparar as medidas das distâncias intercaninos entre sexo e faixas etárias foi realizado o Teste t de Student. Para realizar a comparação entre as distâncias intercaninos, relação molar e más oclusões, foi utilizado o teste ANOVA. Todas as análises foram realizadas utilizando-se o software IBM SPSS versão 25.0, considerando-se um intervalo de confiança de 95\% e um nível de significância de $p<0,05$.

\section{RESULTADOS}

Foram examinadas 110 documentações ortodônticas, de pacientes na faixa etária de 12 a 35 anos de idade de ambos os sexos, pertencentes ao acervo do Curso de Especialização em Ortodontia do Centro Universitário de Patos - UNIFIP/ PB. 
A Tabela 1 descreve a distribuição da amostra segundo as variáveis médias das distâncias intercaninos, idade, sexo e padrão racial. A distância média na arcada superior foi de $33,98 \mathrm{~mm}( \pm 3,04)$, enquanto na arcada inferior esse valor médio correspondeu a $26,11 \mathrm{~mm}( \pm 2,46)$. A média de idade foi de 17,7 anos (DP \pm $5,74)$. A maioria dos pacientes foi do sexo feminino $(58,2 \%)$ e feoderma $(43,6 \%)$.

Tabela 1 - Distribuição da amostra segundo as variáveis: médias das distâncias intercaninos; idade, sexo e padrão racial, $n=110$, Patos-PB, 2020.

\begin{tabular}{lccc}
\hline \multirow{2}{*}{ Arcadas } & \multicolumn{3}{c}{ Distância intercaninos } \\
\cline { 2 - 4 } & Média (DP) & Valor Mínimo $(\mathbf{m m})$ & Valor Máximo $(\mathbf{m m})$ \\
\hline \multicolumn{1}{c}{ Superior } & $33,98( \pm 3,04)$ & 27 & 46 \\
\hline Variáveis & $26,11( \pm 2,46)$ & 19 & 31 \\
\hline Idade & & \\
\hline Média: 17,70 anos & & \\
Desvio Padrão: $\pm 5,74$ & & \\
Valor mínimo: 12 anos & & \\
Valor máximo: 35 anos & $\mathbf{N}$ & \\
\hline Sexo & 64 & \\
\hline Feminino & 46 & 41,8 \\
Masculino & $\mathbf{N}$ & 13,6 \\
\hline Padrão Racial & 15 & 42,7 \\
\hline Melanoderma & 47 & 43,6 \\
Leucoderma & 48 & \\
Feoderma & & & \\
\hline
\end{tabular}

Fonte: Melo TRNB e Souza GR, 2020.

Estão apresentadas na Tabela 2 as variáveis referentes à classificação da relação molar e os tipos de má oclusão. Observou-se, que a maioria dos pacientes tinham a relação molar em Classe I direita $(46,4 \%)$ e esquerda (49,1\%). As menores frequências foram para a Classe III, 16,3\% (direita) e 13,6\% (esquerda). Além disso, $68,2 \%$ da amostra tinha algum tipo de má oclusão. O apinhamento foi a má oclusão mais prevalente na população estudada, correspondendo a $67,7 \%$, sendo a menor frequência para mordida aberta $(7,8 \%)$.

Tabela 2 - Descrição das variáveis referentes à classificação da relação molar e aos tipos de más oclusões, $\mathrm{n}=110$, Patos-PB, 2020.

\begin{tabular}{lcc}
\hline Variáveis & N & $\%$ \\
\hline Relação Molar Direita [110] & & \\
\hline Classe I & 51 & 46,4 \\
Classe II & 41 & 37,3 \\
Classe III & 18 & 16,3 \\
\hline Relação molar Esquerda [110] & 54 & 49,1 \\
\hline Classe I & 41 & 37,3 \\
Classe II & 15 & 13,6 \\
Classe III & & \\
\hline Presença de Má Oclusão [110] & 75 \\
\hline Presente & 35 & 68,2 \\
Ausente & & 31,8 \\
\hline Tipo de Má Oclusão [102] & 08 \\
\hline Mordida aberta & 15 & 7,8 \\
Mordida cruzada anterior & 10 & 14,7 \\
Mordida cruzada posterior & 69 & 9,8 \\
Apinhamento & & 67,7 \\
\hline
\end{tabular}

Fonte: Melo TRNB e Souza GR, 2020. 
A Tabela 3 descreve as associações entre a distância média intercaninos, sexo, faixa etária, relação molar e más oclusões. Observou-se, com diferença significativa $(p=0,01)$, que a distância intercaninos foi maior na arcada superior em pacientes do sexo masculino. Bem como, verificou-se maior distância intercaninos na arcada inferior de pacientes jovens, de 8 a 20 anos, em relação aos adultos, de 21 a 35 anos $(p=0,02)$.

Quanto à relação molar não foi encontrada associação significativa pois a distribuição da amostra foi semelhante entre as classificações $(p>0,05)$. Em relação ao tipo de má oclusão observou-se não haver significância estatística $(p>0,05)$. Pacientes com mordida aberta anterior apresentaram menor distância intercaninos inferior $(25,17 \mathrm{~mm}$; DP $\pm 4,01)$.

Tabela 3 - Associação entre a distância intercaninos, sexo, faixa etária, relação molar e má oclusão, n=110, Patos-PB, 2020, $(p<0,05)$.

\begin{tabular}{|c|c|c|c|c|c|}
\hline \multicolumn{6}{|c|}{ Distância intercaninos } \\
\hline Variáveis & $\mathbf{N}$ & $\begin{array}{c}\text { Superior } \\
\text { Média (DP) }\end{array}$ & $\mathbf{P}$ & $\begin{array}{c}\text { Inferior } \\
\text { Média (DP) }\end{array}$ & $\mathbf{P}$ \\
\hline \multicolumn{6}{|l|}{ Sexo } \\
\hline Feminino & 64 & $33,34( \pm 2,67)$ & \multirow[t]{2}{*}{0,01} & $25,94( \pm 2,29)$ & \multirow[t]{2}{*}{0,41} \\
\hline Masculino & 46 & $34,87( \pm 3,33)$ & & $26,33( \pm 2,68)$ & \\
\hline \multicolumn{6}{|l|}{ Faixa etária } \\
\hline Jovem & 80 & $34,22( \pm 3,11)$ & \multirow[t]{2}{*}{0,18} & $26,42( \pm 2,19)$ & \multirow[t]{2}{*}{0,02} \\
\hline Adulto & 30 & $33,35( \pm 2,81)$ & & $25,27( \pm 2,94)$ & \\
\hline \multicolumn{6}{|l|}{ Relação molar direita } \\
\hline Classe I & 51 & $34,68( \pm 2,96)$ & \multirow{3}{*}{0,06} & $26,52( \pm 2,25)$ & \multirow{3}{*}{0,12} \\
\hline Classe II & 41 & $33,19( \pm 2,95)$ & & $26,01( \pm 2,52)$ & \\
\hline Classe III & 18 & $33,79( \pm 3,17)$ & & $25,16( \pm 2,72)$ & \\
\hline \multicolumn{6}{|l|}{ Relação molar esquerda } \\
\hline Classe I & 54 & $34,06( \pm 3,35)$ & \multirow{3}{*}{0,95} & $26,28( \pm 2,40)$ & \multirow{3}{*}{0,57} \\
\hline Classe II & 41 & $33,95( \pm 2,67)$ & & $26,09( \pm 2,54)$ & \\
\hline Classe III & 15 & $33,78( \pm 3,04)$ & & $25,52( \pm 2,49)$ & \\
\hline \multicolumn{6}{|l|}{ Má oclusão } \\
\hline Mordida aberta anterior & 08 & $34,15( \pm 3,07)$ & \multirow{4}{*}{0,55} & $25,17( \pm 4,01)$ & \multirow{4}{*}{0,59} \\
\hline Mordida cruzada anterior & 12 & $35,20( \pm 4,29)$ & & $25,88( \pm 2,24)$ & \\
\hline Mordida cruzada posterior & 09 & $33,31( \pm 2,81)$ & & $26,46( \pm 1,74)$ & \\
\hline Apinhamento & 55 & $33,98( \pm 2,81)$ & & $26,34( \pm 2,34)$ & \\
\hline
\end{tabular}

Fonte: Melo TRNB e Souza GR, 2020.

\section{DISCUSSÃO}

Durante os tratamentos ortodônticos, onde são necessárias expansões para correção de apinhamentos, mordidas cruzadas ou deficiências maxilares transversais, a alteração em excesso das distâncias intercaninos pode gerar instabilidade e possivelmente recidivas ao término da terapia ortodôntica (KANASHIRO LK, VIGORITO JW, 2007; GUILLEN AGLE, et al., 2020).

Portanto, é importante que sejam obedecidos os diagramas individuais de cada paciente, alterando minimamente essa distância No presente estudo verificou-se que os valores médios das distâncias se mantiveram concordantes com a literatura (INTERLANDI E, 2002; MARQUES JAM, et al, 2005; TRIVIÑO T, et al., 2007; RODRíGEZ AB et al., 2016).

O estudo de Maspero C, et al. (2020), onde foram realizadas as medições das distâncias intercaninos também com paquímetro digital, semelhante à presente pesquisa, foi verificado um aumento na distância intercaninos e intermolares, após utilização de aparelho expansor Hyrax. Entretanto, com o passar do tempo, houve a diminuição dessa distância, demonstrando uma leve recidiva, mesmo em pacientes jovens. 
Essa perda de perímetro é devida à acomodação do próprio organismo, na busca pelo encaixe oclusal, onde o engrenamento entre maxila e mandíbula devem ocorrer pelo contato dentário cúspide/ fossa.

Nos estudos Burke SP, et al. (1998) e Sánchez RMP, et al. (2019) não foram encontradas mudanças na distância intercaninos entre as diferentes classes de más oclusões de Angle, resultado que corrobora com a presente pesquisa. Entretanto, Shapiro PS (1974) e Kanashiro Lk, Vigorito JW (2007), observaram que os pacientes Classe II, $2^{\text {a }}$ divisão, submetidos ao tratamento ortodôntico, possuem uma maior capacidade de expansão da distância intercaninos. Essa capacidade pode estar associada ao fato de que nessa má oclusão os incisivos superiores se encontram lingualizados e os laterais apinhados.

A correção dessas inclinações e angulações dentárias podem contribuir para o aumento do diâmetro da arcada, sem que ocorra vestibularização em excesso, mas sim, que o formato seja devolvido ao seu padrão de normalidade. Esse dado discorda do presente trabalho, onde a distância intercaninos não se correlacionou estatisticamente com pacientes classificados segundo Angle em Classe I, II ou III. Entretanto, seria necessária uma avaliação pós correção ortodôntica para que se descrevesse fidedignamente se há ou não essa correlação.

Marques JAM, et al. (2005) e Sánchez RMP, et al. (2019), estudaram a distância intercaninos em diferentes faixas etárias, correlacionando essas variáveis entre si. Os resultados constataram não haver relação entre tais variáveis. Entretanto, na presente pesquisa, pacientes jovens, de 12 a 20 anos de idade, apresentaram distâncias intercaninos inferiores maiores estatisticamente do que em adultos, de 21 a 35 anos, dados discordantes desses achados. As divergências podem ter ocorrido devido às diferentes amostras e metodologias empregadas. Para a medição do tamanho dos arcos, a discrepância entre os tamanhos mesiodistais dos dentes superiores e inferiores, é considerado um fator determinante (SWEET D, 2005; AL-KHATEEB SN, et al., 2006). Embora não tenha havido, no presente estudo, uma diferença estatisticamente significante no tamanho dos dentes entre os sexos, os dentes nas arcadas masculina foram ligeiramente maiores que nas femininas, enquanto as medidas do arco transversal foram maiores nas mulheres que nos homens, sendo, nesse caso, estatisticamente significante.

Em relação às más oclusões, Perreira JCM, et al. (2011) relataram, em sua pesquisa, que a má oclusão mais prevalente foi a sobremordida profunda. Entretanto, no presente estudo, a maior prevalência foi de apinhamento dentário, seguido das mordidas cruzadas (anterior e posterior), dados que corroboram com o estudo de Patrícia RR, et al. (2007), onde o apinhamento dentário teve também uma maior frequência. Entretanto não houve associação significante com a distância intercaninos, como também em relação às demais oclusopatias estudadas.

A relação molar, segundo a classificação de Angle (Classe I, II e III), também foram verificas na amostra estudada, sendo a maior prevalência observada para a classe I; seguida das classes II e III. Esses achados corroboram com os estudos de Araújo et al. (2014), onde a classe I também apresentou maior incidência dentre a amostra.

Apesar da alta frequência da relação dentária em Classe I de Angle, as más oclusões estudas (mordida aberta, mordida cruzada anterior, mordida cruzada posterior e apinhamento dentário) ocorreram em toda a amostra, demonstrado a necessidade de uma avaliação mais ampla dos arcos dentários e características faciais, visto que, avaliações isoladas, baseadas em modelos de estudo ou medições estáticas, são apenas um indicativo para orientar pesquisas futuras, de abordagem clínica, com aplicação em pacientes.

Diante de todos os pontos elencados e discutidos anteriormente, fica clara a necessidade de outras pesquisas que enfoquem a distância intercaninos e a correlacione com outras variáveis, como as más oclusões, visto que, mais estudos são necessários para se somar a literatura clássica e atual.

\section{CONCLUSÃO}

De acordo com a literatura consultada e a metodologia empregada na presente pesquisa, pode-se concluir que os valores das distâncias intercaninos mantiveram-se semelhantes às relatadas na literatura, bem como não houve associação significante entre essas distâncias e as más oclusões. Os estudos 
observacionais possuem como limitação a falta de intervenção clínica. Entretanto, pesquisas dessa natureza podem ser um guia para futuros ensaios clínicos.

\section{REFERÊNCIAS}

1. AL-KHATEEB SN, ABU ALHAIJA ES. Tooth size discrepancies and arch parameters among different malocclusions in a Jordanian sample. Angle Orthod, 2006, 76 (3): 459-465.

2. ANDREWS LF. The six keys to normal occlusion. American journal of Orthodontics, 1972; 62: $296-309$.

3. ANGLE, E. Treatment of malocclusion of the teeth. Angle's system. 7th ed. Philadelphia: S. S. White Manufacturing, 1907.

4. ARAÚJO SCCS, et al. Análise da força de mordida nos diferentes tipos de maloclusões dentárias, segundo Angle. Rev. CEFAC. 2014; 16(5):1567-1578.

5. BONDEVIK O. Differences between high and low angle subjects in arch form and anterior crowding from 23 to 33 years of age. Eur J Orthod, 2007; 29(4): 413-416.

6. BURKE SP, et al. A meta-analysis of mandibular intercanine width in treatment and post retention. Angle Orthod, 1998; 68(1): 53-60.

7. GUILLÉN ALE, et al. Inter-premolar width changes related to the orthodontic traction of maxillary impacted canines in adolescents and young adults: A retrospective CBCT study. Int Orthod. 2020; 28 (3): 22.

8. HENRIKSON J, et al. Long-term stability of dental arch form in normal occlusion from 13 to 31 years of age. Eur $\mathrm{J}$ Orthod, 2001; 23(1): 51-61.

9. INTERLANDI S. Diagrama de contorneamento ortodôntico para a técnica do arco contínuo (Straight Wire). Ortodontia, 2002; 35 (1) 91-105.

10. KANASHIRO LK, VIGORITO JW. Distância entre as faces vestibulares dos arcos dentários e o rebordo alveolar em diferentes tipos de oclusão. Ortodontia, 2007; 40(2): 115-124.

11. MARQUES JAM, et al. Estudo da distância intercanina no processo de identificação de marcas de mordidas. Rev Assoc Paul Cir Dent, 2005; 59: 363-6.

12. MASPERO C, et al. Hyrax versus transverse sagittal maxillary expander: An assessment of arch changes on dental casts. A retrospective study. Saudi Dent J, 2020; 32(2): 93-100.

13. PATRICIA RR, et al. Avaliação das alterações na distância intercaninos do arco inferior em pacientes com má oclusão de Classe I tratados ortodonticamente. Rev. Dental Press Ortodon Ortop Facial, 2007; 12(2): 232-241.

14. PEREIRA JCM, et al. Estudo comparativo cefalométrico dos padrões dentofaciais de indivíduos portadores de oclusão normal e de más oclusões de Angle. Rev. Dental Press J Orthod, 2011; 16 (5): 62-73.

15. RODRÍGEZ BA, et al. Forma y tamaño del arco dental en poblaciones de tres ascendencias étnicas colomianas. Ces Odontol, 2016, 29 (2): 20-32.

16. SÁNCHEZ RMP, et al. Arch parameters and dental discrepancy (crowding and spacing) in a sample of an AfroColombian population. Acta Odontol Latinoam, 2019, 32 (2): 88-96.

17. SHAPIRO PS. Mandibular dental arch form and dimension: treatment and post retention changes. Am $\mathrm{J}$ Orthod, 1974; 66(1): 58-70.

18. SOUSA JP, SOUSA SA. Prevalência de má oclusão em escolares de 7 a 9 anos de idade do Polo 1 da Rede Municipal de Ensino em João Pessoa-PB. Rev Odontol UNESP, 2013; 42(2): 117-123.

19. SWEET D. Marks from teeth as forensic evidence - the state of the art. Revista Aragonesa de Medicina Legal, 2005; 7: 91-98.

20. TRIVIÑO T, et al. A forma do arco dentário inferior na visão da literatura. Rev. Dental Press Ortodon Ortop Facial, $2007 ; 12(6): 61-72$. 